\title{
SEMANTIC Processing MECHANism For LISTENING AND COMPREHENSION IN VNSCALENDAR SYSTEM
}

\author{
Thien Khai $\operatorname{Tran}^{1} \&$ Dang Tuan Nguyen ${ }^{2}$ \\ ${ }^{1}$ Natural Language and Knowledge Engineering Research Group, University of \\ Information Technology, Vietnam National University - Ho Chi Minh City, Vietnam. \\ tkthienenlke-group. net \\ ${ }^{2}$ Faculty of Computer Science, University of Information Technology, Vietnam National \\ University - Ho Chi Minh City, Vietnam. \\ dangnteuit.edu.vn
}

\section{ABSTRACT}

This paper presents some generalities about the VNSCalendar system, a tool able to understand users' voice commands, would help users with managing and querying their personal calendar by Vietnamese speech. The main feature of this system consists in the fact that it is equipped with a mechanism of analyzing syntax and semantics of Vietnamese commands and questions. The syntactic and semantic processing of Vietnamese sentences is solved by using DCG (Definite Clause Grammar) and the methods of formal semantics. This is the first system in this field of voice application, which is equipped an effective semantic processing mechanism of Vietnamese language. Having been built and tested in PC environment, our system proves its accuracy attaining more than $91 \%$.

\section{KEYWORDS}

Speech Recognition, Voice Application, Vietnamese, Natural Language Processing, Formal Semantics.

\section{INTRODUCTION}

In 2012, Vietnam saw many remarkable publications displayed by groups devoting to spoken Vietnamese recognition researches from Institute of Information Technology (Vietnamese Academy of Science and Technology) and University of Science, VNU-HCM. It is worth mentioning the works of Thang $\mathrm{Vu}$ and Mai Luong [13] as well as Quan Vu et al. [1], [3], [5], [9]. The authors crucially concentrated on improving the efficiency of their voice recognition system, such as the Quan Vu et al. 's one which obtained the precision rate of over than $93 \%$ and this group successfully built many voice applications on this base. Nevertheless, all the applications have not been accompanied with a efficient semantic processing mechanism yet, which is the important mechanism in view of helping the system with understanding commands.

In this paper, we introduce the VNSCalendar system. It is a tool, as a combination of the spoken language recognition and the written language processing, would help users with managing and querying their personal calendar by Vietnamese speech commands. Our system can recognize many forms of Vietnamese speech commands and questions, convert them into text, resolve their syntax and semantic analysis, then, generate database queries, and finally, return the results to 
user. The work of resolving syntactic parsing and semantic analysis of Vietnamese commands and question is based on using DCG [2], [7] and computational techniques of formal semantics [6], [10], [11].

In this research, we focus on semantic processing of Vietnamese commands and questions. We only deal with a Vietnamese speech recognition task by using HTK (Hidden Markov Model Toolkit) [12] and build a training data as well as testing data for it.

\section{SYSTEM ARCHITECTURE}

In accordance with Thien Khai Tran [14], our system is designed to carry out these functions as bellow:

1. Add events: add events into the calendar by Vietnamese speech.

2. Delete events: remove events out of the calendar by Vietnamese speech.

3. Edit events: edit events from the calendar by Vietnamese speech.

4. Query events: query events from the calendar by Vietnamese speech.

5.

VNSCalendar fulfills these above functions in observing the further scenario:

The interaction between users and system can be presented in brief as following steps:

Step 0 Listening stage

Step 1 User says to the system by Vietnamese.

Step 2 The speech sentence is converted into the Vietnamese text sentence thanks to the Speech Recognizer.

Step 3 The system analyzes the syntax structure and gets the key information of the text sentence.

(3.1) If the input sentence is a command:

If it is an add command:

- The system adds the associated event to the schedule calendar and confirms the result to user.

- Return Step 0.

If it is a delete command:

- The system deletes the associated event from the schedule calendar and confirms the result to user.

- $\quad$ Return Step 0.

If it is an edit command:

- The system deletes the event needing to edit and adds the associated event to the schedule calendar and then confirms the result to user.

- $\quad$ Return Step 0.

(3.2) If the input sentence is a query

- The system executes the query, searches information in the database and shows the result to user.

- Return Step 0.

(3.3) In case the syntax is incorrect, the system will inform user of it and user can take another 
command/query.

To realize the functions in observing the above scenario, the system must be composed of following components:

1. Automatic speech recognizer (ASR): identify words that user speaks, then convert them into written text.

2. Vietnamese language processor: resolve the syntax and semantic representations of all the command sentences or query sentences of user.

3. Central processor:

- Transform the semantic representations of the command / query sentences into the SQL commands and execute it.

- Filter, organize, and return the results to user.

4. Database: store schedule information

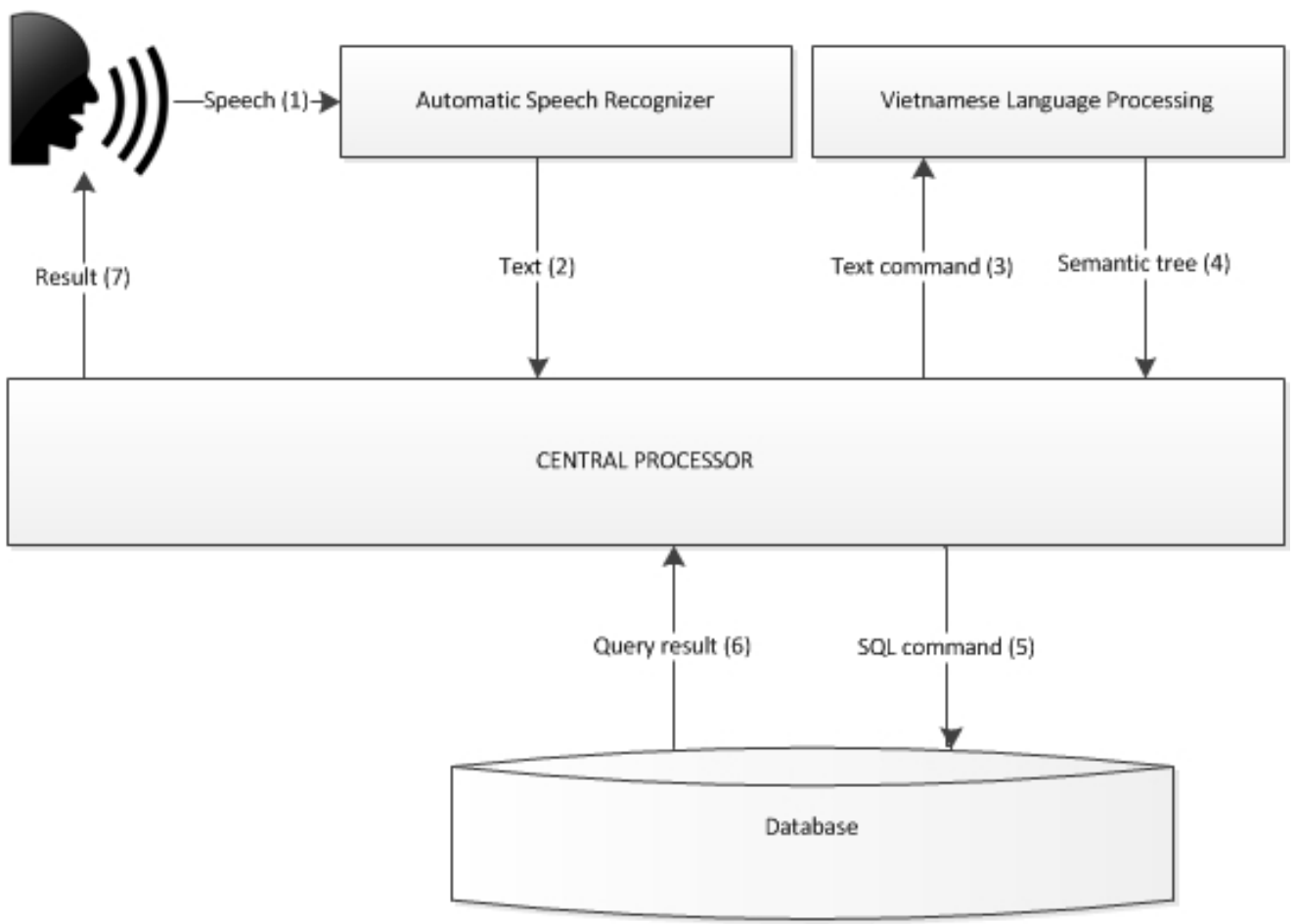

Figure 1. Architecture of VNSCalendar ( Source in [Thien Khai Tran 2013] )

\section{Automatic SPEech ReCOGNIZER}

In VNSCalendar system, we have used HTK [12] to build the Automatic Speech Recognition component. According to the approach of Quan Vu et al. [9], we have applied the contextdependent model based on triphone [12] to recognize keywords and grammar terms. 


\subsection{Training Data}

The speech corpus has 1,045 sentences. Total audio training covers 77 minutes. All speech was sampled at $16000 \mathrm{~Hz}, 16$ bit by PCM format in a relatively quiet environment with a single speaker.

The lexical comprises of 96 keywords and 19 grammar terms as shown in Table 1 and Table 2 . There are some compound words that we cannot translate their extracted single word meaning.

Table 1. List of keywords (Source: Thien Khai Tran [14])

\begin{tabular}{|c|c|c|c|c|c|c|c|}
\hline $\begin{array}{c}\text { ăn } \\
\text { (eat) }\end{array}$ & $\begin{array}{c}\text { ba } \\
\text { (three) }\end{array}$ & $\begin{array}{c}\text { bài } \\
\text { (lesson) }\end{array}$ & $\begin{array}{c}\mathrm{b} \mathrm{n} \\
\text { (friend) }\end{array}$ & $\begin{array}{c}\text { b y } \\
\text { (seven) }\end{array}$ & $\begin{array}{c}\text { báo } \\
\text { (newspaper) }\end{array}$ & $\begin{array}{l}\mathrm{b} n \\
\text { (four) }\end{array}$ & $\begin{array}{c}\text { bu i } \\
\text { (session) }\end{array}$ \\
\hline cà & cáo & $\begin{array}{c}\text { chi u } \\
\text { (evening) }\end{array}$ & $\begin{array}{l}\text { chín } \\
\text { (nine) }\end{array}$ & $\begin{array}{c}\text { ch ng } \\
\text { (husband) }\end{array}$ & ch & $\begin{array}{l}\text { con } \\
\text { (child) }\end{array}$ & công \\
\hline $\begin{array}{l}\mathrm{c} m \\
\text { (rice) }\end{array}$ & $\begin{array}{l}\text { cùng } \\
\text { (with) }\end{array}$ & $\begin{array}{l}\mathrm{cu} \mathrm{i} \\
\text { (end) }\end{array}$ & $\begin{array}{c}\text { d y } \\
\text { (teach) }\end{array}$ & $\begin{array}{c}\text { đ u } \\
\text { (early) }\end{array}$ & đ & $\begin{array}{l}\text { đi } \\
\text { (go) }\end{array}$ & di $n$ \\
\hline $\begin{array}{c}\text { đ c } \\
(\mathrm{read})\end{array}$ & đ ng & $\begin{array}{l}\text { đón } \\
\text { (get) }\end{array}$ & $\mathrm{du}$ & $\begin{array}{c}\mathrm{d} \\
\text { (attend) }\end{array}$ & $\underset{\text { (visit) }}{\mathrm{g} \mathrm{p} p}$ & $\begin{array}{l}\text { gi } \\
\text { (hour) }\end{array}$ & $\begin{array}{l}\text { hàng } \\
\text { (goods) }\end{array}$ \\
\hline $\begin{array}{l}\text { hai } \\
\text { (two) }\end{array}$ & $\begin{array}{c}\text { h c } \\
\text { (study) }\end{array}$ & $\begin{array}{c}\mathrm{h} p \\
\text { (meeting) }\end{array}$ & h i & hôm & khách & $\begin{array}{l}\text { làm } \\
\text { (do) }\end{array}$ & $\begin{array}{c}1 \mathrm{ch} \\
\text { (calendar) }\end{array}$ \\
\hline $\begin{array}{c}1 \mathrm{p} \\
\text { (class) }\end{array}$ & $\begin{array}{c}\text { mai } \\
\text { (tomorrow) }\end{array}$ & $\begin{array}{c}\mathrm{m} \\
\text { (mother) }\end{array}$ & $\begin{array}{l}\mathrm{m} \mathrm{t} \\
\text { (one) }\end{array}$ & $\begin{array}{c}\mathrm{m} \mathrm{t} \\
\text { (the day } \\
\text { after } \\
\text { tomorrow) }\end{array}$ & $\begin{array}{l}\mathrm{m} i \\
\text { (ten) }\end{array}$ & $\mathrm{m} \quad \mathrm{i}$ & $\begin{array}{l}\text { này } \\
\text { (this) }\end{array}$ \\
\hline $\begin{array}{l}\text { năm } \\
\text { (year) }\end{array}$ & $\begin{array}{l}\text { nay } \\
\text { (this) }\end{array}$ & $\begin{array}{l}\text { ngày } \\
\text { (day) }\end{array}$ & $\begin{array}{l}\text { nghe } \\
\text { (listen) }\end{array}$ & nghi $\mathrm{p}$ & $\begin{array}{c}\text { ng } \quad \mathrm{i} \\
\text { (person) }\end{array}$ & $\begin{array}{c}\mathrm{nh} \mathrm{n} \\
\text { (receive) }\end{array}$ & nh t \\
\hline $\begin{array}{c}\mathrm{nh} \mathrm{u} \\
\text { (drinking) }\end{array}$ & $\begin{array}{c}\text { nhà } \\
\text { (home) }\end{array}$ & $\begin{array}{c}\text { nh c } \\
\text { (music) }\end{array}$ & (in) & phê & $\begin{array}{l}\text { phim } \\
\text { (film) }\end{array}$ & $\begin{array}{c}\text { phút } \\
\text { (minute) }\end{array}$ & $\begin{array}{c}\text { qu n } \\
\text { (district) }\end{array}$ \\
\hline $\begin{array}{l}\text { sách } \\
\text { (book) }\end{array}$ & $\begin{array}{c}\text { sáng } \\
\text { (morning) }\end{array}$ & $\begin{array}{l}\text { sáu } \\
\text { (six) }\end{array}$ & $\begin{array}{c}\text { sau } \\
\text { (next) }\end{array}$ & $\begin{array}{c}\text { s p } \\
\text { (boss) }\end{array}$ & $\begin{array}{l}\mathrm{t} p \\
\text { (do) }\end{array}$ & tài & $\begin{array}{l}\text { t i } \\
\text { (at) }\end{array}$ \\
\hline tác & $\begin{array}{l}\text { tám } \\
\text { (eight) }\end{array}$ & $\begin{array}{l}\text { th } \mathrm{u} \\
\text { (bid) }\end{array}$ & $\begin{array}{c}\text { th } y \\
\text { (teacher) }\end{array}$ & $\begin{array}{l}\text { thân } \\
\text { (relative) }\end{array}$ & thao & th 0 & $\begin{array}{l}\text { tháng } \\
\text { (month) }\end{array}$ \\
\hline th & $\begin{array}{c}\text { thi } \\
\text { (exam) }\end{array}$ & th & $\begin{array}{c}\text { ti c } \\
\text { (party) }\end{array}$ & $\begin{array}{c}\mathrm{t} \mathrm{i} \\
\text { (night) }\end{array}$ & $\begin{array}{c}\mathrm{t} \mathrm{i} \\
(\text { next) }\end{array}$ & $\begin{array}{c}\operatorname{tr} \mathrm{a} \\
\text { (afternoon) }\end{array}$ & $\begin{array}{l}\text { tr ng } \\
\text { (school) }\end{array}$ \\
\hline $\begin{array}{c}\text { tu n } \\
\text { (week) }\end{array}$ & $\begin{array}{c}\mathrm{t} \\
\text { (fourth) }\end{array}$ & ty & $\begin{array}{l}\text { u ng } \\
\text { (drink) }\end{array}$ & văn & $\begin{array}{c}\mathrm{v} \\
(\text { wife })\end{array}$ & $\begin{array}{c}\mathrm{v} \text { i } \\
\text { (with) }\end{array}$ & $\begin{array}{c}\text { xem } \\
\text { (watch) }\end{array}$ \\
\hline
\end{tabular}


International Journal on Natural Language Computing (IJNLC) Vol. 2, No.2, April 2013

Table 2. List of grammar terms (Source: Thien Khai Tran [14])

\begin{tabular}{|c|c|c|c|c|}
\hline $\begin{array}{c}\text { ai } \\
\text { (who) }\end{array}$ & có & gì & hay & khi \\
\hline $\begin{array}{c}\text { không } \\
\text { (yes or not) }\end{array}$ & $\begin{array}{c}\text { lúc } \\
\text { (at/ in/ on) }\end{array}$ & đâu & nào & nh \\
\hline $\begin{array}{c}\mathrm{s} \text { a } \\
\text { (edit) }\end{array}$ & $\begin{array}{c}\text { t o } \\
\text { (create) }\end{array}$ & $\begin{array}{c}\text { thành } \\
\text { (become) }\end{array}$ & $\begin{array}{c}\text { thay } \\
\text { (edit) }\end{array}$ & th \\
\hline $\begin{array}{c}\text { thêm } \\
\text { (add) }\end{array}$ & $\begin{array}{c}\text { vào } \\
\text { (to) }\end{array}$ & $\begin{array}{c}\text { vô } \\
\text { (into) }\end{array}$ & $\begin{array}{c}\text { xóa } \\
\text { (delete) }\end{array}$ & \\
\hline
\end{tabular}

\subsection{Steps to build the Automatic Speech Recognizer}

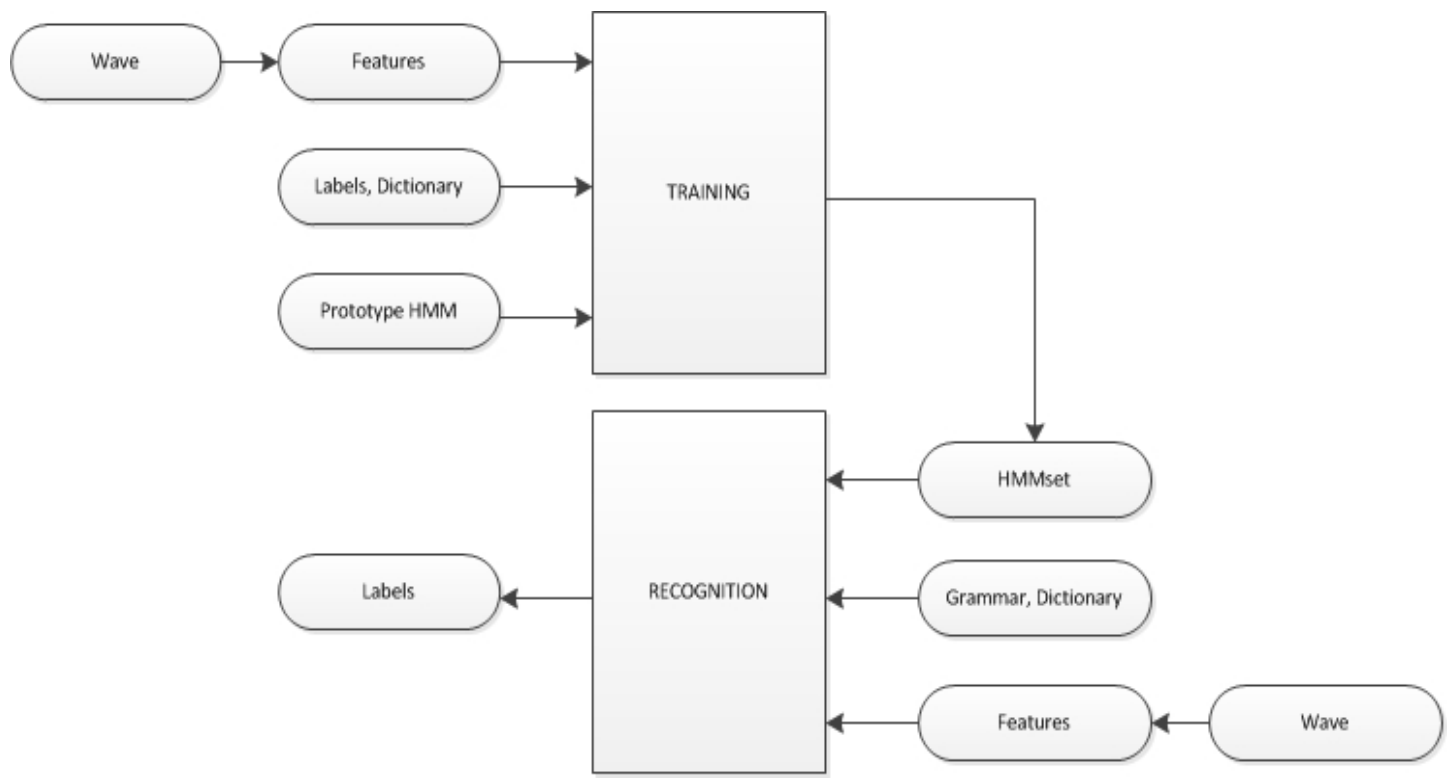

Figure 2. Steps to build the Automatic Speech Recognizer. (Source: Thien Khai Tran [14])

\section{Vietnamese Language Processing}

The Vietnamese language processing component analyzes syntax and semantics of Vietnamese commands and questions. Semantic processing aims at computing the semantic structures of the commands or questions. These semantic structures are presented by FOL (First-Order Logic) [2], [7]. In this research, we use techniques of computational semantics [6], [10], [11].

\subsection{Commands}

The semantic structures of Add, Del, Edit commands are listed in Table 3, Table 4, Table 5. 
International Journal on Natural Language Computing (IJNLC) Vol. 2, No.2, April 2013

Table 3. Semantic structures of Add-command (Source: Thien Khai Tran [14])

\begin{tabular}{|l|l|}
\hline 1 & add(time,work(action,obj)) \\
\hline 2 & add(time,work(action,obj),place) \\
\hline 3 & add(time,work(action,obj),person) \\
\hline 4 & add(time,work(action,obj),person,place) \\
\hline
\end{tabular}

Example 1: Thêm $\mathrm{s}$ ki $\mathrm{n} d$ th u $\mathrm{t}$ i công ty lúc chín gi th ba tu $\mathrm{n}$ sau. (Add event of bidding at the company at 9 am next Tuesday)

The syntactic and semantic rules in DCG are defined as below:

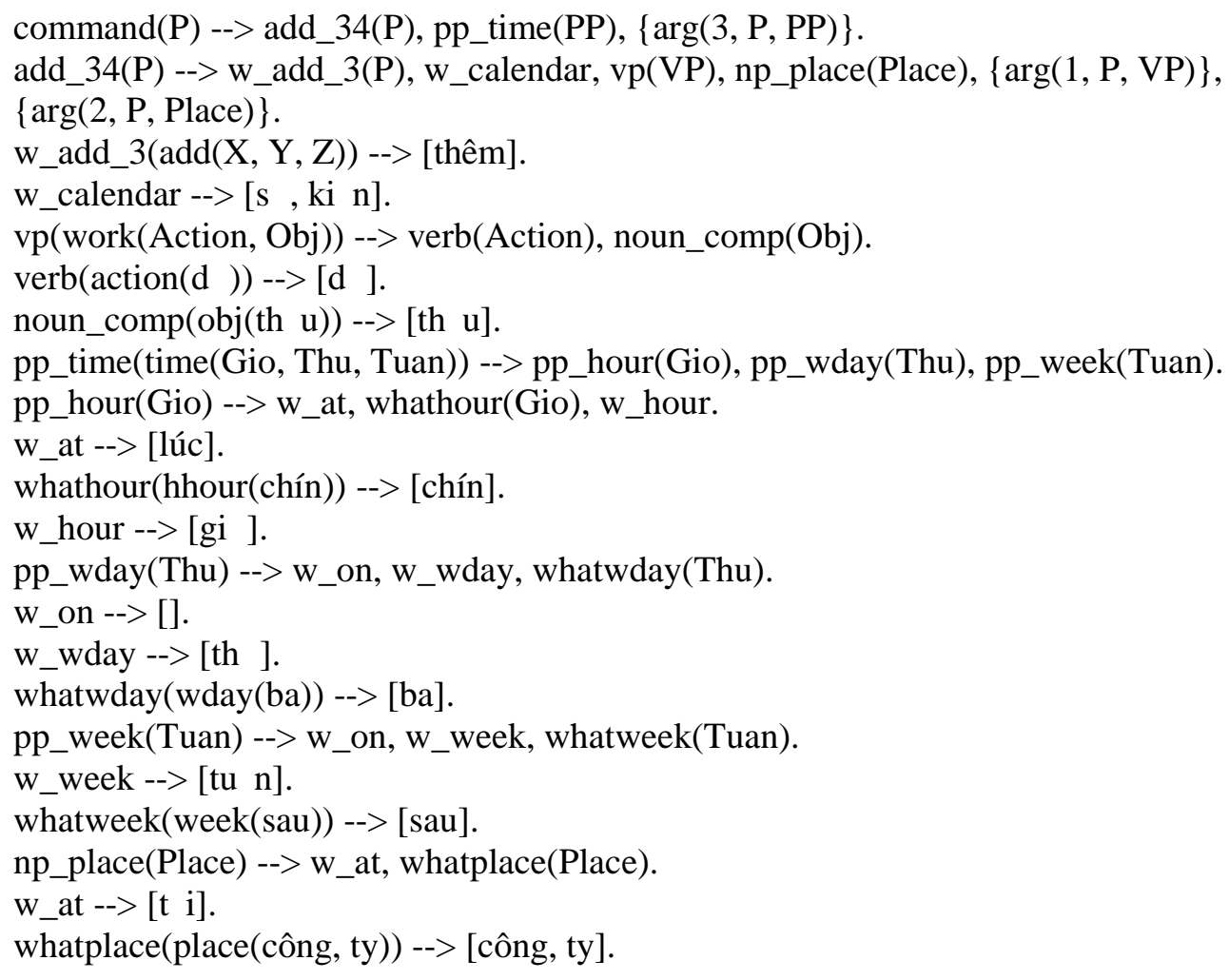

These syntactic and semantic rules determine the semantic structure of this command as below: add(time(hour(chín), wday(ba), week(sau)), work(action(d ), obj(th u)), place(công ty)).

This semantic structure is the structure 2 in Table 3. 
Table 4. Semantic structures of Del-command (Source: Thien Khai Tran [14])

\begin{tabular}{|c|l|}
\hline 5 & del(time,work(action,obj)) \\
\hline 6 & del(time,work(action,obj),place) \\
\hline 7 & del(time,work(action,obj),person) \\
\hline 8 & del(time,work(action,obj),person,place) \\
\hline
\end{tabular}

Example 2: Lo i b kh i l ch $\mathrm{m}$ i ba gi ngày hai tám tháng $\mathrm{m}$ i hai báo cáo đ tài. (Delete event of reporting topic at 13 o'clock on December 28th)

The syntactic \& semantic rules in DCG are defined as below:

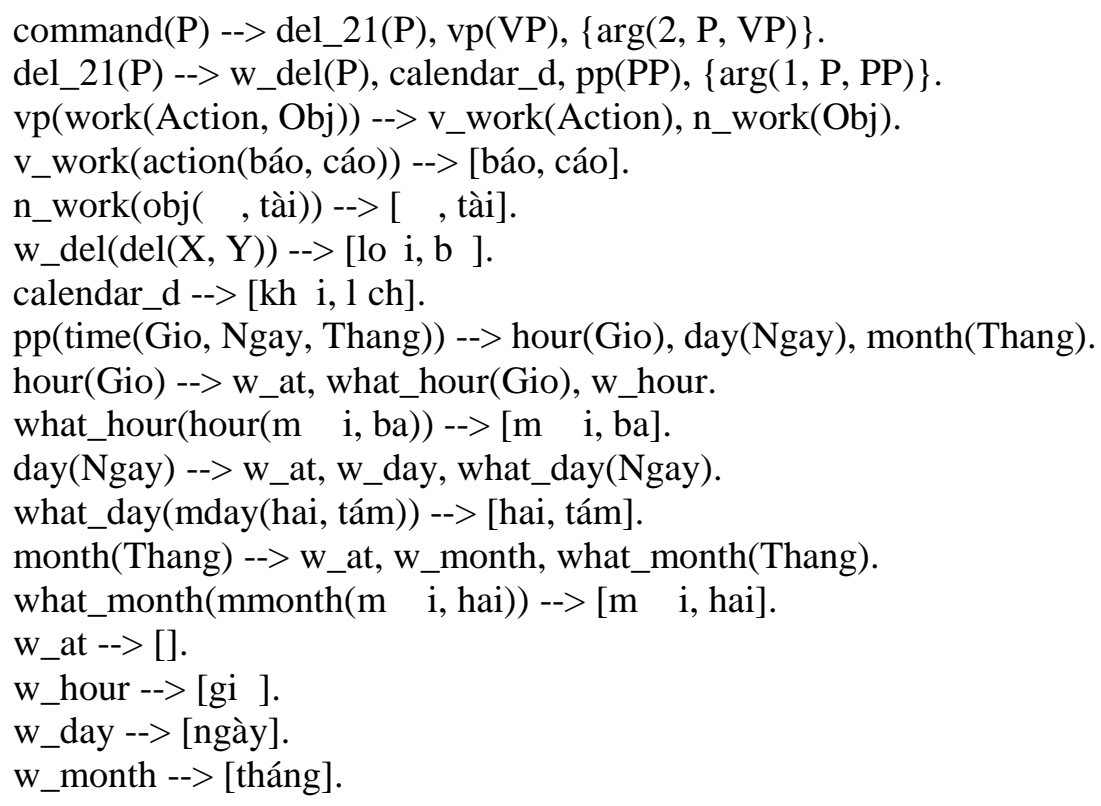

These syntactic and semantic rules determine the semantic structure of this command as below:

del(time(hour(m i, ba), mday(hai, tám), mmonth(m i, hai)), work(action(báo, cáo), $\operatorname{obj}(đ$, tài $)))$

This semantic structure is the structure 5 in Table 4 . 
Table 5. Semantic structures of Edit-command (Source: Thien Khai Tran [14])

\begin{tabular}{|c|c|}
\hline 9 & edit(del ( time, work(action, obj)), add(time, work(action, obj))) \\
\hline 10 & edit(del(time, work(action, obj)), add(time, work(action, obj), person)) \\
\hline 11 & edit(del(time, work(action, obj)), add(time, work(action, obj), place)) \\
\hline 12 & edit(del(time,work(action, obj)), add(time, work(action, obj), person, place)) \\
\hline 13 & edit(del(time,work(action, obj), person), add(time, work(action, obj)) \\
\hline 14 & edit(del(time,work(action, obj), person), add(time, work(action, obj), person) \\
\hline 15 & edit(del(time,work(action, obj), person), add(time, work(action, obj), place) \\
\hline 16 & edit(del(time,work(action, obj), person), add(time, work(action, obj), person, place) \\
\hline 17 & edit(del(time,work(action, obj), place), add(time, work(action, obj)) \\
\hline 18 & edit(del(time,work(action, obj), place), add(time, work(action, obj), person) \\
\hline 19 & edit(del(time,work(action, obj), place), add(time, work(action, obj), place) \\
\hline 20 & edit(del(time,work(action, obj), place), add(time, work(action, obj), place) \\
\hline 21 & edit(del(time,work(action, obj), person, place), add(time, work(action, obj)) \\
\hline 22 & edit(del(time,work(action, obj), person, place), add(time, work(action, obj), person) \\
\hline 23 & edit(del(time, work(action, obj), person, place), add(time, work(action, obj), place) \\
\hline 24 & edit(del(time, work(action, obj), person, place), add(time, work(action, obj), person, place) \\
\hline
\end{tabular}

Example 3: Ch nh $1 \mathrm{i} d$ th u $\mathrm{t}$ i công ty ngày $\mathrm{m}$ i tám tháng chín thành đi công tác ngày $\mathrm{m}$ i tám tháng chín. (Adjust bidding at the company on September $18^{\text {th }}$ by business traveling on September $18^{\text {th }}$ )

The syntactic and semantic rules determine the semantic structure of this command as below:

del(time(hour(m i, ba), mday(hai, tám), mmonth(m i, hai)), work(action(báo, cáo), $\operatorname{obj}(đ$, tài $))$ )

This semantic structure is the structure 17 in Table 5 .

\subsection{Questions}

The semantic structures of questions forms are listed in Table 6, Table 7, Table 8, Table 9, Table 10, Table 11, Table 12.

Table 6. Semantic structures of Yes/No question (Source: Thien Khai Tran [14]) 


\begin{tabular}{|c|l|}
\hline 1 & yesno(time, action, obj) \\
\hline 2 & yesno(time, action, obj, place) \\
\hline 3 & yesno(time, action, obj, person) \\
\hline 4 & yesno(time, action, obj, person, place) \\
\hline
\end{tabular}

Example 4: Mai có đi h c không? (Going to school tomorrow or not?)

The syntactic and semantic rules determine the semantic structure of this question as below: yesno(time(dday(mai)), action(đi), obj(h c)).

This semantic structure is structure 1 in Table 6.

Table 7. Semantic structures of WHAT question (Source: Thien Khai Tran [14])

5 workQuery(query(action), query(obj), time)

Example 5: Cu i tu n sau có làm gì không? (Any job for next weekend?)

The syntactic \& semantic rules in DCG are defined as below:

query(workQuery(Action, Obj, Time)) --> pp_time(Time), interrogative1, verb_query (Action), noun_query (Obj), interrogative2.

pp_time(time(Tuan)) --> pp_week(Tuan).

pp_week(week(Prep, Tuan)) --> w_at, pp_prep(Prep), w_week, whatweek(Tuan).

pp_prep(prep(cu i)) --> [cu i ] .

w_week --> [tu n].

whatweek(week(sau)) --> [sau].

interrogative 1 --> [có].

interrogative2 --> [không].

verb_query(query(action)) --> w_do.

noun_query(query(obj)) --> w_what.

W_do --> [làm].

W_what --> [gì].

These syntactic and semantic rules determine the semantic structure of this question as below:

workQuery(query(action), query(obj), time(prep(cu i), week(sau))).

This semantic structure is the structure 5 in Table 7. 
International Journal on Natural Language Computing (IJNLC) Vol. 2, No.2, April 2013

Table 8. Semantic structures of Go_WHERE question (Source: Thien Khai Tran [14])

\begin{tabular}{|c|l|}
\hline 6 & gowhereQuery(query(obj), verb_go, time) \\
\hline 7 & gowhereQuery(query(obj), verb_go, person, time) \\
\hline 8 & gowhereQuery(query(obj), verb_go, place, time) \\
\hline 9 & gowhereQuery(query(obj), verb_go, person, place, time) \\
\hline
\end{tabular}

Example 6: Tháng m i đi đâu nh ? (Where to go in October?)

The syntactic and semantic rules determine the semantic structure of this question as below:

gowhereQuery(query(obj), verb_go(đi), time(mmonth(m i $)$ )).

This semantic structure is the structure 6 in Table 8 .

Table 9. Semantic structures of Visit_WHOM question (Source: Thien Khai Tran [14])

\begin{tabular}{|l|l|}
\hline 10 & visitwhomQuery(query(obj), verb_visit, time) \\
\hline 11 & visitwhomQuery(query(obj), verb_visit, person, time) \\
\hline 12 & visitwhomQuery(query(obj), verb_visit, place, time) \\
\hline 13 & $\begin{array}{l}\text { visitwhomQuery(query(obj), verb_visit, person, place, } \\
\text { time) }\end{array}$ \\
\hline
\end{tabular}

Example 7: Tu n sau có g p ai không nh ?(Whom to meet next week?)

The syntactic and semantic rules determine the semantic structure of this question as below:

visitwhomQuery(query(obj),verb_visit(g p),time(week(sau)))

This semantic structure is the structure 10 in Table 9.

Table 10. Semantic structures of With_WHOM question (Source: Thien Khai Tran [14])

\begin{tabular}{|l|l|}
\hline 14 & personQuery(query(person),action,obj,time) \\
\hline 15 & personQuery(query(person),action,obj,place,time) \\
\hline
\end{tabular}

Example 8: $\mathrm{M}$ t báo cáo đ tài v i ai?

The syntactic and semantic rules determine the semantic structure of this question as below:

personQuery(query(person),action(báo, cáo),obj(đ , tài),time(dday(m t))). 
This semantic structure is the structure 14 in Table 10.

Table 11. Semantic structures of WHERE question (Source: Thien Khai Tran [14])

\begin{tabular}{|l|l|}
\hline 16 & placeQuery(query(place),action,obj,time) \\
\hline 17 & placeQuery(query(place),action,obj,person,time) \\
\hline
\end{tabular}

Example 9: M t báo cáo đ tài v i b $\mathrm{n}$ đâu?

The syntactic and semantic rules determine the semantic structure of this question as below:

placeQuery(query(place), action(báo, cáo),obj(đ , tài), person(b n), time(dday(m t))).

This semantic structure is the structure 17 in Table 11.

Table 12. Semantic structures of WHEN question (Source: Thien Khai Tran [14])

\begin{tabular}{|l|l|}
\hline 18 & timeQuery(query(time),action,obj) \\
\hline 19 & timeQuery(query(time),action,obj,person) \\
\hline 20 & timeQuery(query(time), action,obj,person,place) \\
\hline
\end{tabular}

Example 10: Đi du 1 ch khi nào? (When to travel?)

The syntactic and semantic rules determine the semantic structure of this question as below: timeQuery(query(time), action(đi),obj(du $1 \mathrm{ch})$ ).

This semantic structure is the structure 18 in Table 12 .

\subsection{Pragmatic Semantics Processing}

This research also carries out timing situations in relation to pragmatic semantics in sentences. All of timing units will be formatted in a standard timing structure: "yyyy:mm:dd hh:mm".

Table 13. Hypothesis for pragmatic semantics of timing

\begin{tabular}{|l|l|l|}
\hline Keyword & Context & Pragmatic Semantics \\
\hline \multirow{3}{*}{$\begin{array}{l}\text { Đ u } \\
\text { (beginning of) }\end{array}$} & $\begin{array}{l}\text { đ u tu n } \\
\text { (beginning of week) }\end{array}$ & Monday $\rightarrow$ Tuesday \\
\cline { 2 - 3 } & $\begin{array}{l}\text { đ u tháng } \\
\text { (beginning of month) }\end{array}$ & day $1 \rightarrow$ day 10 of month \\
\cline { 2 - 3 } $\begin{array}{l}\text { đ u năm } \\
\text { (beginning of year) }\end{array}$ & January $\rightarrow$ March \\
\hline
\end{tabular}


International Journal on Natural Language Computing (IJNLC) Vol. 2, No.2, April 2013

\begin{tabular}{|c|c|c|}
\hline \multirow{3}{*}{$\begin{array}{l}\mathrm{Cu} \mathrm{i} \\
\text { (end of) }\end{array}$} & $\begin{array}{l}\text { cu i tu } \mathrm{n} \\
\text { (end of week) }\end{array}$ & Saturday $\rightarrow$ Sunday \\
\hline & $\begin{array}{l}\text { cu i tháng } \\
\text { (end of month) }\end{array}$ & day $20 \rightarrow$ day 31 of month \\
\hline & $\begin{array}{l}\text { cu i năm } \\
\text { (end of year) }\end{array}$ & month $10 \rightarrow 12$ of year \\
\hline \multirow{3}{*}{$\begin{array}{l}\mathrm{Sau}=\mathrm{T} \mathrm{i} \\
(\text { next })\end{array}$} & $\begin{array}{l}\text { tu n sau } \\
\text { (next week) }\end{array}$ & next Monday $\rightarrow$ next Sunday \\
\hline & $\begin{array}{l}\text { tháng sau } \\
\text { (next month) }\end{array}$ & $\begin{array}{l}\text { current month (recording } \\
\text { time) }+1\end{array}$ \\
\hline & $\begin{array}{l}\text { năm sau } \\
\text { (next year) }\end{array}$ & $\begin{array}{l}\text { current year (recording time) } \\
+1\end{array}$ \\
\hline $\begin{array}{l}\text { Sáng } \\
\text { (morning) }\end{array}$ & & $4 \mathrm{am} \rightarrow 10 \mathrm{am}$ \\
\hline $\begin{array}{l}\operatorname{Tr} \mathrm{a} \\
\text { (noon) }\end{array}$ & & $10 \mathrm{am} \rightarrow 15 \mathrm{pm}$ \\
\hline $\begin{array}{l}\text { Chi u } \\
\text { (evening) }\end{array}$ & & $15 \mathrm{pm} \rightarrow 18 \mathrm{pm}$ \\
\hline $\begin{array}{l}\mathrm{T} \text { i } \\
\text { (night) }\end{array}$ & & $18 \mathrm{pm} \rightarrow 24 \mathrm{am}$ \\
\hline $\begin{array}{l}\text { Mai } \\
\text { (tomorrow) }\end{array}$ & $\begin{array}{l}\text { ngày mai } \\
\text { (tomorrow) }\end{array}$ & current day + 1 \\
\hline $\begin{array}{l}\mathrm{M} \mathrm{t} \\
\text { (the day after } \\
\text { tomorrow) }\end{array}$ & $\begin{array}{l}\text { Ngày } \mathrm{m} \mathrm{t} \\
\text { (the day after } \\
\text { tomorrow) }\end{array}$ & current day +2 \\
\hline
\end{tabular}

Table 14. Examples of schedule time prediction (Source: Thien Khai Tran [14])

\begin{tabular}{|c|c|c|c|c|c|c|}
\hline \multirow[t]{2}{*}{ Recording time } & \multicolumn{5}{|c|}{ Timing phrase } & \multirow[t]{2}{*}{ Prediction time } \\
\hline & prep & session & day & week & month & \\
\hline \multirow{4}{*}{$\begin{array}{l}\text { Th 5, } \\
\text { Ngày 13-9-2012 } \\
\text { (Sep.13, 2012) }\end{array}$} & & $\begin{array}{l}\text { sáng } \\
\text { (morning) }\end{array}$ & $\begin{array}{l}\text { mai } \\
\text { (tomorrow) }\end{array}$ & & & $\begin{array}{l}\text { 4h } \rightarrow \text { 10h 14/9/2012 } \\
(4 a m-10 a m \text { Sep. } \\
14,2012)\end{array}$ \\
\hline & & & & $\begin{array}{l}\text { sau } \\
\text { (next) }\end{array}$ & & $\begin{array}{l}\mathrm{t} \quad 17 / 9 \rightarrow 23 / 9 \\
(\text { Sep. } 17 \rightarrow \text { Sep. } 19)\end{array}$ \\
\hline & $\begin{array}{l}\text { cu i } \\
\text { (end } \\
\text { of) }\end{array}$ & & & & $\begin{array}{l}\mathrm{t} \mathrm{i} \\
\text { (next) }\end{array}$ & $\begin{array}{l}\mathrm{t} \quad 20 / 10 \rightarrow 31 / 10 \\
\text { (Oct. } 20 \rightarrow \text { Oct. } 31)\end{array}$ \\
\hline & & & & $\begin{array}{l}\text { này } \\
\text { (this) }\end{array}$ & & $\begin{array}{l}10 / 9 \rightarrow 16 / 9 \\
(\text { Sep. } 10 \rightarrow \text { Sep. } 16)\end{array}$ \\
\hline
\end{tabular}


International Journal on Natural Language Computing (IJNLC) Vol. 2, No.2, April 2013

Table 15. Examples of timing part of commands and questions (Source: Thien Khai Tran [14])

\begin{tabular}{|l|l|l|}
\hline Command sentence & Schedule time & Query sentence \\
\hline $\begin{array}{l}\text { 8g sáng th 3 tu n sau đi h c } \\
\text { (8am next Tuesday, going to } \\
\text { school) }\end{array}$ & $\begin{array}{l}\text { 8h ngày 18/9/2012 } \\
\text { (8am Sep. 18, 2012) }\end{array}$ & $\begin{array}{l}\text { Ngày 18-9 có đi h c không? } \\
\text { (Going to school on Sept. 18, or } \\
\text { not?) }\end{array}$ \\
\hline $\begin{array}{l}\text { G p khách hàng 15g30 ngày m t } \\
\text { (visiting partner at 15.30pm the } \\
\text { day after tomorrow) }\end{array}$ & $\begin{array}{l}\text { 15h ngày 15/9/2012 } \\
\text { (15am Sep. 15, 2012) }\end{array}$ & $\begin{array}{l}\text { Chi u ngày 15 có làm gì không? } \\
\text { (Any job for the evening of 15?) }\end{array}$ \\
\hline $\begin{array}{l}\text { Ngày 28-10 đi du 1 ch v i nhà } \\
\text { (On Oct, 28 travelling with } \\
\text { family) }\end{array}$ & $\begin{array}{l}\text { Ngày 28-10 } \\
\text { (Oct. 28) }\end{array}$ & $\begin{array}{l}\text { Cu i tháng sau có đi đâu không? } \\
\text { (Going anywhere by the end of } \\
\text { next month?) }\end{array}$ \\
\hline
\end{tabular}

Example 11a: Thêm s ki $\mathrm{n} d$ th u $\mathrm{t}$ i công ty lúc chín gi th ba tu $\mathrm{n}$ sau. (Add event of bidding at the company at 9 am next Tuesday)

The semantic structure: add(time(“2012:09:25 09:00”), action(d ), obj(th u), place(công ty)). Example 11b: $\mathrm{Cu}$ i tu $\mathrm{n}$ sau có làm gì không? (Any job for next weekend?)

The semantic structure: workQuery(query(action), query(obj), time(“2012:09:29 00:00”“2012:09:30 00:00”))

The SQL command generated by above semantic structure will query all records in database with "time" field satisfying: "2012:09:29 00:00" stime s"2012:09:30 00:00".

\section{EXPERIMENTS AND EVALUATION}

As mentioned in Thien Khai Tran [14], we have separately carried out tests the two components: Automatic Speech Recognizer and Vietnamese Language Processing. Afterwards, the synthetic experimental step of whole system has achieved

\subsection{Speech Recognizer}

\subsubsection{Evaluation Score}

The speech recognition performance is typically evaluated in terms of Word Error Rate (WER), which can then be computed as: $\mathrm{WER}=(\mathrm{S}+\mathrm{D}+\mathrm{I}) / \mathrm{N} \times 100 \%$ [12], where $\mathrm{N}$ is the total number of words in the testing data, $\mathrm{S}$ denotes the total number of substitution errors, $\mathrm{D}$ is the total number of deletion errors and $\mathrm{I}$ is the total number of insertion errors.

We make use of Word Accuracy (WA) [12] instead, which is computed as WA $=(1-(\mathrm{S}+\mathrm{D}+\mathrm{I})$ / N) x $100 \%$, to report performance of the speech recognizer. 


\subsubsection{Performance}

We have valued the accuracy of the Vietnamese speech recognizer component with 60 sentences, a single speaker in a relatively quiet environment. The results prove that WA can reach $98,09 \%$ and average time processing is 1.4 seconds/ sentence.

There are two main reasons explaining that high score: 1- This is a single speaker-dependent speech recognition, 2- We have used context-dependent model based on triphone for speech recognizer component, and made a strict grammar rules for the recognizer.

\subsection{Vietnamese Language Processing}

We have done manual tests including 60 sentences for evaluating the performance of the Vietnamese processing component. They are pattern sentences found in 48 semantic structures which have built in view of the system. The latter is capable of handling all the pattern sentences.

\subsection{System Experiments}

The system has been built as a PC-based application by MS Visual C\# 2010 and SWI-Prolog version 6.2.1 for Windows NT/2000/XP/Vista/7.

Table 16. Experimental Environments

\begin{tabular}{|l|l|}
\hline Number of Commands & 38 \\
\hline Number of Questions & 22 \\
\hline Environment & in-door \\
\hline Sampling rate & $16 \mathrm{kHz}$ \\
\hline Quantization & $16 \mathrm{bits}$ \\
\hline Format & PCM \\
\hline
\end{tabular}

The system correctly analyzes and executes 55/60 of the spoken commands in Vietnamese language. The fault cases must be remained at the speech recognition step. So, our system demonstrates its accuracy attaining more than $91 \%$. About 2.8 seconds for a command is spent as the average feedback time of the system.

We have also evaluated the capacity of handling the semantic commands of the system by using other approaches - keyword matching, phrase matching, such as longest matching algorithm used by Quan Vu et al. [9].

The results showed the 39 tested sentences out of 60 incorrect (65\%). That proves the considerable improvement of the correctness of VNSpeechCalender system in handling the semantic commands and questions. 


\section{CONCLUSION}

This paper has presented the architectural model of VNSCalendar system as well as our approach to build it. The Vietnamese Laguaguage Processing component, which can analyze syntax and semantics of some Vietnamese speech commands and questions forms, is centered on this system. With this research, we have provided evidence of the importance of syntax and semantics processing in voice applications, specially Vietnamese speech applications. In next steps, our jobs to be accomplished have essential characters in executing an independent speech recognition and widen vocabulary to realize the application as well as to develop simillar applications based on this research background.

\section{REFERENCES}

[1] Duong Dau, Minh Le, Cuong Le and Quan Vu, (2012). “A Robust Vietnamese Voice Server for Automated Directory Assistance Application”. RIVF-VLSP 2012. Ho Chi Minh City, Viet Nam.

[2] Fernando C. N. Pereira and Stuart M. Shieber, (2005). Prolog and Natural-Language Analysis. Microtome Publishing, Massachusetts.

[3] Hue Nguyen, Truong Tran, Nhi Le, Nhut Pham and Quan Vu, (2012). "iSago: The Vietnamese Mobile Speech Assistant for Food-court and Restaurant Location”. RIVF-VLSP 2012. Ho Chi Minh City, Viet Nam.

[4] Jan Wielemaker, (2012). SWI-Prolog version 6.2.2. Internet: www.swi-prolog.org. [Now. 22, 2012].

[5] Nhut Pham and Quan Vu, (2010). "A Spoken Dialog System for Stock Information Inquiry". in Proc. IT@EDU. Ho Chi Minh City, Viet Nam.

[6] Patrick Blackburn, Johan Bos, (2007). Representation and Inference for Natural Language: A First Course in Computational Semantics. CSLI Press, Chicago.

[7] Pierre M. Nugues, (2006). An Introduction to Language Processing with Perl \& Prolog. SpringerVerlag, Berlin.

[8] Po-Chuan-Lin, (2010). "Personal Speech Calendar with Timing Keywords Aware and Schedule Time Prediction Functions". TENCON 2010 IEEE, Japan, pp. 746 - 750.

[9] Quan Vu et al., (2012). "Nghiên c u xây d ng h th ng Voice Server và ng $\mathrm{d}$ ng cho các $\mathrm{d}$ ch v tr 1 it đ ng qua đi $n$ tho i”. Technical report, Research project, HCM City Department of Science and Technology, Viet Nam.

[10] Richard Montague (1974). Formal Philosophy: Selected Papers of Richard Montague. Bell \& Howell Information \& Lea, New Haven.

[11] Sandiway Fong, (2006). LING 364: Introduction to Formal Semantics. www.dingo.sbs.arizona.edu/ sandiway. [Nov. 22, 2012].

[12] Steve Young et al., (2006). The HTK Book (version 3.4). [On-line]. Available: www.htk.eng.cam.ac.uk/docs/docs.shtml [Nov. 1, 2012].

[13] Thang Vu and Mai Luong, (2012). "The Development of Vietnamese Corpora Toward Speech Translation System". RIVF-VLSP 2012. Ho Chi Minh City, Viet Nam.

[14] Thien Khai Tran, (2013). "Xây d ng công c tr giúp qu n lý và truy v n 1 ch cá nhân b ng ti ng nói”. Master's thesis. Faculty of Computer Science, University of Information Technology, Vietnam National University - Ho Chi Minh City, Viet Nam. 